\title{
Abnormal Hemodynamics in the Pulmonary Artery Seen on Time-Resolved 3-Dimensional Phase-Contrast Magnetic Resonance Imaging (4D-Flow) in a Young Patient With Idiopathic Pulmonary Arterial Hypertension
}

Keiichi Odagiri, MD, PhD; Naoki Inui, MD, PhD; Sachiko Miyakawa, MD, PhD; Akio Hakamata; Jiazhang Wei; Yasuo Takehara, MD, PhD; Harumi Sakahara, MD, PhD; Masataka Sugiyama; Marcus T. Alley; Quang-Kim Tran, MD, PhD; Hiroshi Watanabe, MD, PhD
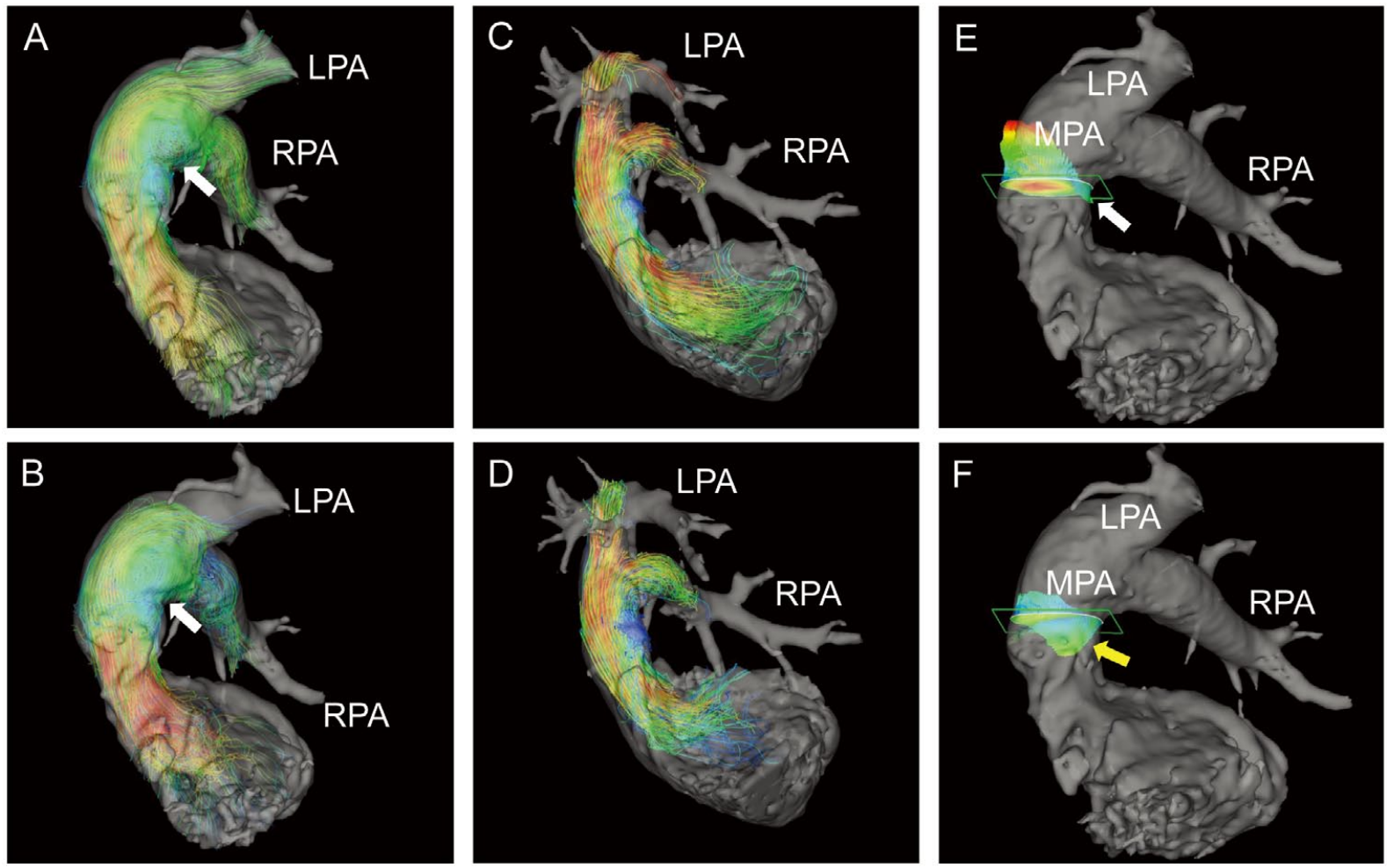

Figure 1. (A,B) Three-dimensional (A) streamline and (B) pathline visualization of blood flow during systole in the pulmonary artery (PA) in a pulmonary arterial hypertension (PAH) patient. (C,D) For comparison, 3-D streamline in a healthy volunteer is also shown (C, streamline; D, pathline). White arrows, vortex blood flow; blue, $0 \mathrm{~mm} / \mathrm{s}$; red, maximum blood velocity. (E,F) Main PA antegrade and retrograde flow during $(E)$ early and $(F)$ late systole. White arrow, early systolic retrograde flow corresponding to the dorsal side of the PA; yellow arrow, end-systolic retrograde flow that occurred with pulmonary valve closure. LPA, left pulmonary artery; MPA, main pulmonary artery; RPA, right pulmonary artery.

Received March 6, 2014; accepted April 2, 2014; released online April 30, 2014 Time for primary review: 13 days

Department of Clinical Pharmacology and Therapeutics (K.O., N.I., S.M., A.H., J.W., H.W.), Department of Radiology (Y.T., H.S., M.S.), Hamamatsu University School of Medicine, Hamamatsu, Japan; Department of Radiology, Stanford University, Palo, Alto, CA (M.T.A.); and Department of Physiology and Pharmacology, Des Moines University, Des Moines, IA (Q.-K.T.), USA

Mailing address: Keiichi Odagiri, MD, PhD, Department of Clinical Pharmacology and Therapeutics, Hamamatsu University School of Medicine, 1-20-1 Handayama, Higashi-ku, Hamamatsu 431-3192, Japan. E-mail: kodagiri@ hama-med.ac.jp

ISSN-1346-9843 doi:10.1253/circj.CJ-14-0283

All rights are reserved to the Japanese Circulation Society. For permissions, please e-mail: cj@j-circ.or.jp 

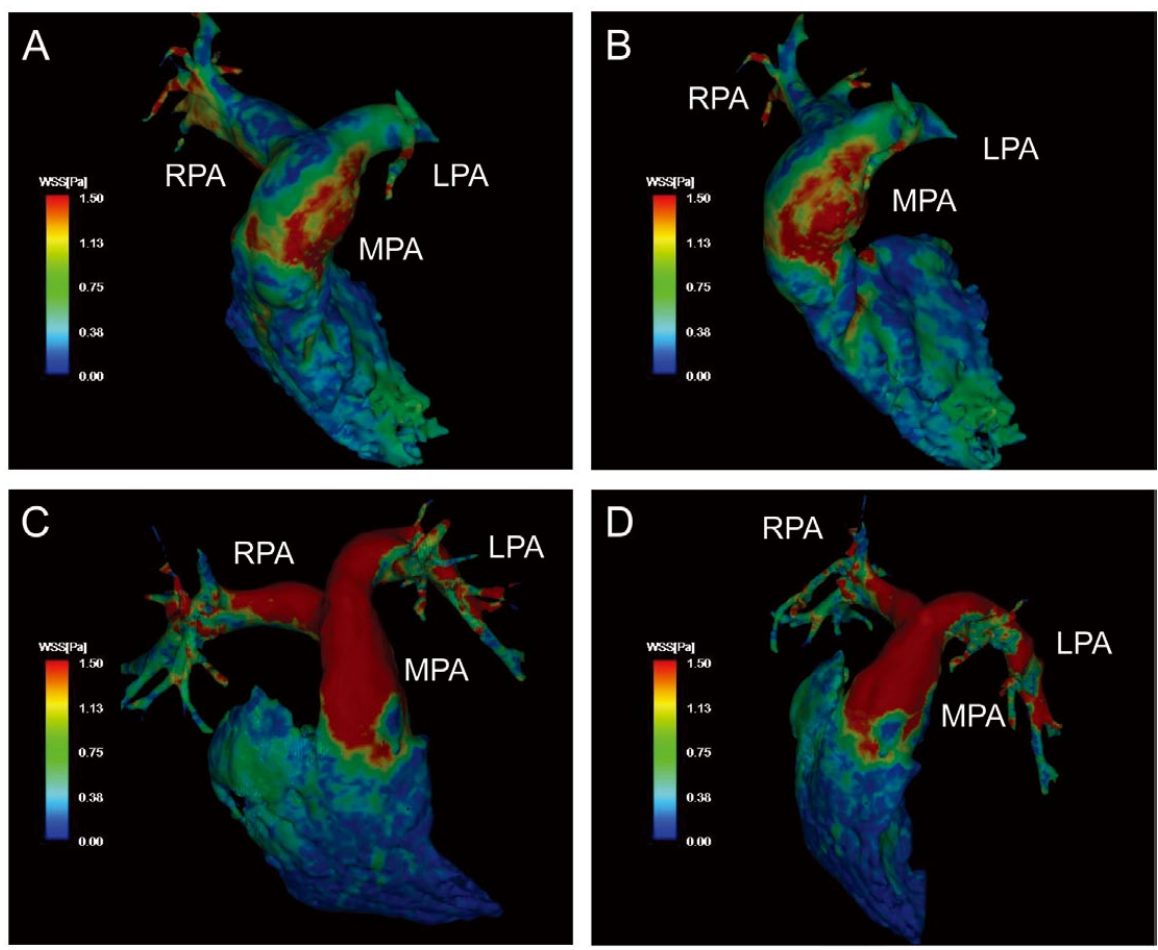

Figure 2. Three-dimensional visualization of wall shear stress (WSS) during peak systole in the (A) right anterior oblique (RAO) and (B) left anterior oblique (LAO) view. (C,D) For comparison, 3-D visualization of WSS in a healthy volunteer is also shown (C, RAO view; D, LAO view). The pulmonary artery wall color indicates WSS: the color shift from blue to red indicates increased WSS. Blue, $0 \mathrm{~Pa}$; red, >1.5 Pa. LPA, left pulmonary artery; MPA, main pulmonary artery; RPA, right pulmonary artery.

A 16-year-old woman presented to a local city hospital for evaluation of shortness of breath and electrocardiographic abnormality. Twelve-lead echocardiogram (ECG), lung perfusion scintigraphy and right heart catheterization supported the diagnosis of idiopathic pulmonary arterial hypertension (PAH). After discussion with her cardiologist, she consulted Hamamatsu University Hospital for further treatment.

She had World Health Organization functional assessment classification class II symptoms of dyspnea on effort. Cardiac auscultation indicated a III/VI tricuspid regurgitation (TR) murmur and a loud P2 component of S2 heart sound. ECG showed normal sinus rhythm with right-axis deviation, complete right bundle branch block and right ventricular hypertrophy. Echocardiography showed right ventricular hypertrophy without dilatation (right ventricular diameter, $34.9 \mathrm{~mm}$ measured on apical 4-chamber view), severe TR with high right ventricle systolic pressure $105 \mathrm{mmHg}$ (estimated using TR pressure gradient), but normal left ventricular function (left ventricular ejection fraction, $79.7 \%$ ). Cardiac catheterization measurements included mean pulmonary artery (PA) pressure $(84 \mathrm{mmHg} ; 125 / 57 \mathrm{mmHg})$, mean right atrial pressure $(10 \mathrm{mmHg})$, mean pulmonary capillary wedge pressure $(13 \mathrm{mmHg})$, and cardiac output $(7.75 \mathrm{~L} / \mathrm{min}$; cardiac index, $5.11 \mathrm{~L} \cdot \mathrm{min}^{-1} \cdot \mathrm{m}^{-2}$ determined on thermodilution).

To further characterize the patient's pulmonary arterial hemodynamics, gadolinium-enhanced magnetic resonance angiography and phase-resolved 3-dimensional (3-D) phase-contrast magnetic resonance imaging (MRI; 4D-flow) of the PA was done. These techniques allow non-invasive identification of blood flow patterns, measurement of blood flow velocities and wall shear stress (WSS) at each cardiac phase. Whole heart 3-D MRI showed dilatation of the PA. The 3-D streamline (Figure 1A; Movie S1) and pathline (Figure 1B) images clearly demonstrated the existence of vortex blood flow in the main PA, whereas this blood flow pattern was not seen in a healthy volunteer (Figures 1C,D). The duration of vortex was $0.27 \mathrm{~s}$ (30.0\% of the cardiac cycle). Maximum and mean blood flow velocities at the main PA were $0.45 \mathrm{~m} / \mathrm{s}$ and $0.07 \mathrm{~m} / \mathrm{s}$, respectively. Cross-sectional flow analysis indicated biphasic retrograde flow in the main PA (Figures 1E,F). The location of the early systolic retrograde flow corresponded to the dorsal side of the PA. Three-dimensional surface analysis indicated that the WSS was spatially and temporally heterogeneous (Figures 2A,B; Movie S2) compared with a healthy volunteer (Figures 2C,D). Maximum and mean WSS at the central PA were 0.95 Pa and $0.44 \mathrm{~Pa}$, respectively.

In PAH, cardiac MRI is a useful method for assessing right ventricular function. Right ventricular volume parameters obtained on cardiac MRI are predictors of hospitalization and mortality. ${ }^{1}$ Phase-contrast cardiac MRI can also be used to evaluate blood flow volumes and velocities, and visualize blood flow patterns., ${ }^{2,3}$ Previous studies have shown that PA blood flow pattern in $\mathrm{PAH}$ patients differs from that in non-PAH participants. ${ }^{2}$ The vortex blood flow appearance and the early onset of retrograde flow in main PA are believed to be characteristic of PAH. ${ }^{2,4,5}$ The duration of vortex is closely related to the mean PA pressure. ${ }^{2,5}$ It was reported that WSS in PAH patients is significantly decreased, which might be a parameter that reflects the progression of the disease. ${ }^{6}$ WSS is decreased 
when abnormal flow dynamics such as vortex or turbulent flow occur adjacent to the arterial wall. ${ }^{7,8}$ Therefore, low WSS on the PA wall reflects abnormal hemodynamics occurring within the PA. Previous investigators also reported that the decreased WSS inhibited nitric oxide release from the endothelium. ${ }^{9-11}$ Color-coded 3-D WSS readily demonstrates PA flow abnormalities and resultant decreased WSS on the PA in a time-resolved and quantitative fashion. Abnormal hemodynamics may also be shown on streamline analysis, but only qualitatively. The present report shows that 4D-flow is a useful noninvasive method for the qualitative and quantitative characterization of not only blood flow but also WSS in PAH patients.

\section{Acknowledgments}

K.O. has received research funding from Japan Research Foundation for Clinical Pharmacology and lecture fees from Pfizer and Acterion. H.W. has received research funding from the Ministry of Health, Labour and Welfare of Japan, Teika Seiyaku, Takeda Pharmaceuticals, Mochida, Pfizer, Astellas Pharmaceuticals and Daiichi Sankyo, and lecture fees from Pfizer, Acterion, Novartis, Daiichi Sankyo, GlaxoSmithKline and Nippon Shinyaku. These funders had no role in the study design, data collection and analysis, decision to publish, or preparation of the manuscript. The other authors state that they have no conflict of interest.

\section{Sources of Funding}

None.

\section{References}

1. Yamada Y, Okuda S, Kataoka M, Tanimoto A, Tamura Y, Abe T, et al. Prognostic value of cardiac magnetic resonance imaging for idiopathic pulmonary arterial hypertension before initiating intravenous prostacyclin therapy. Circ J 2012; 76: 1737-1743.

2. Reiter G, Reiter U, Kovacs G, Kainz B, Schmidt K, Maier R, et al. Magnetic resonance-derived 3-dimensional blood flow patterns in the main pulmonary artery as a marker of pulmonary hypertension and a measure of elevated mean pulmonary arterial pressure. Circ Cardiovasc Imaging 2008; 1: 23-30.

3. Sugimoto M, Kajino H, Kajihama A, Nakau K, Murakami N, Azuma $\mathrm{H}$. Assessment of pulmonary arterial pressure by velocity-encoded cine magnetic resonance imaging in children with congenital heart disease. Circ J 2013; 77: 3015-3022.

4. Helderman F, Mauritz GJ, Andringa KE, Vonk-Noordegraaf A, Marcus JT. Early onset of retrograde flow in the main pulmonary artery is a characteristic of pulmonary arterial hypertension. J Magn Reson Imaging 2011; 33: 1362-1368.

5. Reiter U, Reiter G, Kovacs G, Stalder AF, Gulsun MA, Greiser A, et al. Evaluation of elevated mean pulmonary arterial pressure based on magnetic resonance 4D velocity mapping: Comparison of visualization techniques. PLoS One 2013; 8: e82212, doi:10.1371/journal. pone.0082212.

6. Tang BT, Pickard SS, Chan FP, Tsao PS, Taylor CA, Feinstein JA. Wall shear stress is decreased in the pulmonary arteries of patients with pulmonary arterial hypertension: An image-based, computational fluid dynamics study. Pulm Circ 2012; 2: 470-476.

7. Burk J, Blanke P, Stankovic Z, Barker A, Russe M, Geiger J, et al. Evaluation of 3D blood flow patterns and wall shear stress in the normal and dilated thoracic aorta using flow-sensitive 4D CMR. $J$ Cardiovasc Magn Reson 2012; 14: 84.

8. Prado CM, Rossi MA. Aorta remodeling responses to distinct atherogenic stimuli: Hypertension, hypercholesterolemia and turbulent flow/low wall shear stress. Open Cardiovasc Med J 2008; 2: 41-48.

9. Corson MA, James NL, Latta SE, Nerem RM, Berk BC, Harrison DG. Phosphorylation of endothelial nitric oxide synthase in response to fluid shear stress. Circ Res 1996; 79: 984-991.

10. Fisher AB, Chien S, Barakat AI, Nerem RM. Endothelial cellular response to altered shear stress. Am J Physiol Lung Cell Mol Physiol 2001; 281: L529-L533.

11. Noris M, Morigi M, Donadelli R, Aiello S, Foppolo M, Todeschini $\mathrm{M}$, et al. Nitric oxide synthesis by cultured endothelial cells is modulated by flow conditions. Circ Res 1995; 76: 536-543.

\section{Supplementary Files}

Supplementary File 1

Movie S1. Three-dimensional streamline visualizations of blood flow in the pulmonary artery. The appearance of a vortex of blood flow is visualized.

Supplementary File 2

Movie S2. Three-dimensional visualization of wall shear stress on the central pulmonary artery.

Please find supplementary file(s);

http://dx.doi.org/10.1253/circj.CJ-14-0283 